\title{
The Use of A Mobile Sink for Quality Data Collection in Energy Harvesting Sensor Networks
}

\author{
Xiaojiang Ren \\ Weifa Liang \\ Research School of Computer Science \\ Australian National University \\ Canberra, ACT 0200, Australia \\ Email: richard.rxj@anu.edu.au,wliang@cs.anu.edu.au
}

\begin{abstract}
In this paper we study data collection in an energy harvesting sensor network where sensors are deployed along a given path and a mobile sink travels along the path periodically for data collection. Such a typical application scenario is to employ a mobile vehicle for traffic surveillance of a given highway. As the sensors in this network are powered by renewable energy sources, the time-varying characteristics of energy harvesting poses great challenges on the design of efficient routing protocols for data collection in harvesting sensor networks. In this paper we first formulate a novel optimization problem as a network utility maximization problem, by incorporating multi-rate communication mechanism between sensors and the mobile sink and show the NP-hardness of the problem. We then devise a novel centralized algorithm for it, assuming that the global knowledge of the entire network is available. We also develop a distributed solution to the problem without the global knowledge assumption. We finally conduct extensive experiments by simulations to evaluate the performance of the proposed algorithms. The experimental results demonstrate that the proposed algorithms are promising and very efficient.
\end{abstract}

\section{INTRODUCTION}

Wireless sensor network has emerged as a key technology for various applications such as environmental sensing, structural health monitoring, and area surveillance. Regardless of energy efficiency of battery-powered sensor networks (referred to as conventional sensor networks), they will fail eventually due to the depletion of power resource. In some harsh environments, replacing batteries will be very costly and sometimes become impossible. A viable solution against the limited energy supplies is to enable sensor nodes to harvest ambient energy from their surroundings. However, the timevarying characteristics of energy harvesting sources poses great challenges in the design of routing protocols for such networks under the dynamic energy replenishment constraints.

\section{A. Related work}

Sink mobility in conventional sensor networks has been extensively studied [2], [3], [6], [11], [12], [13], [18], [21], [22], [23], and shown that it can reduce the energy consumption of sensors, balance the workload among sensors, thereby prolonging network lifetime. Most existing studies focused on minimizing the energy consumption so as to prolong network lifetime since sensors are powered by energy-limited batteries. The proposed approaches for conventional sensor networks however are not applicable to energy harvesting sensor networks, due to the fact that the time-varying energy replenishment is imposed on the latter. In other words, the network lifetime metric in energy harvesting sensor networks is no longer a major issue as the energy powering sensors can be replenished periodically. Thus, routing protocols for energy harvesting sensor networks should be adaptive to response to dynamic changes of energy replenishment rates.

In terms of data collection with a path-constrained mobile sink, the closely related work in conventional sensor networks is briefly described as follows. Song and Hatzinakos [18] considered the energy consumption minimization problem of data collection from one-hop sensors. They formulated the problem as a joint power control and time allocation optimization problem by providing a Markov-chain model. Chakrabarti et al. [2] considered the dependence of transmission setting and packet loss rate of the mobile data collection problem by modeling the process of data collection as a M/D/1 queue. They then proposed an algorithm that ensures adequate data collection and minimizes the energy consumption. Kansal et al. [9], [19] addressed a network infrastructure based on the use of a path-constrained mobile sink for data collection, where a sensor sends its data to the sink along a minimum number hop-count path. They then proposed a speed control algorithm to improve the amount of data collected. Assuming that the mobile sink moves at a constant speed, Gao et al. [6] addressed the energy minimization problem by proposing a novel data collection scheme, where sensors close to the trajectory of the mobile sink are chosen as subsinks and other sensors make use of different subsinks for their data relay. They formulated the subsink choice problem as the problem of minimizing the total sum of hops from sensors to their subsinks and provided a heuristic algorithm. They then studied the time allocation problem for subsinks by divided the communication time between the mobile sink and all subsinks into time intervals and proposed some practical time allocation methods. In contrast, little attention has been paid to data collection in energy harvesting sensor networks with mobile sinks [16]. Most existing studies on data collection in such networks assumed that the collected data is routed to a fixed sink through multi-hop relays, which may not be applicable to large-scale networks [5], [10], [14], [24]. For 
example, Liu et al. [10], [14] formulated the problem as a lexicographic maximin rate allocation problem, and provided a centralized algorithm for the problem by solving an integer program. Zhang et al. [24] studied the problem as a utility maximization problem by representing the utility gain at each sensor node as a concave utility function. They proposed an efficient algorithm for finding the accumulative sum of utility gains of all sensors in tree networks. Orthogonal to these existing works, in this paper we consider data collection in an energy harvesting sensor network with a path-constrained mobile sink, where the sensor network is deployed along a highway for traffic-surveillance and a mobile vehicle at a constant speed is used to patrol the highway for collecting data from its one-hop sensors. We formulate the problem as a network utility maximization problem by incorporating multirate wireless communication mechanism between the sensors and the mobile sink.

\section{B. Contribution}

Our major contributions in this paper are as follows. We consider data collection in an energy harvesting sensor network with a path-constrained mobile sink. We first formulate the problem as a novel network utility maximization problem and show its NP-hardness. We then propose a centralized algorithm assuming that the global knowledge of the entire network is available. We also develop a distributed algorithm for the problem without the global knowledge. Finally, we conduct experimental evaluation by simulations to evaluate the performance of the proposed algorithms. The experimental results demonstrate that the proposed algorithms are efficient.

\section{Paper organization}

The remainder of the paper is organized as follows. Section II introduces the system model, notions, problem definition, and its NP-hardness proof. Section III is devoted to devising a centralized algorithm for the network utility maximization problem, and a distributed algorithm is also proposed in Section IV. Section V evaluates the performance of the proposed algorithms through experimental simulations, and Section VI concludes the paper.

\section{Preliminaries}

\section{A. System model}

We consider an energy harvesting sensor network $G=$ $(V \cup\{s\}, E)$ where $V$ is a set of $n$ homogeneous sensors deployed along a path and $s$ is a mobile sink traveling along the path at a constant speed to collect data from one-hop sensors. Each sensor is powered by solar energy and has stored enough sensing data for collection. There is a link in $E$ between a sensor $v \in V$ and $s$ when $s$ is within the transmission range of $v$.

Given the path length $L$, the tour time of the mobile sink per tour is determined by its speed (or the data latency requirement). That is, the faster the mobile sink moves, the shorter its tour time is, resulting in a shorter delay on data delivery. We here consider a discrete-time system where the duration per tour is slotted into equal time slots with each lasting $\tau$ time units [15]. Given the mobile sink speed $r_{s}$, the number of time slots can be determined by $T=\left\lceil\frac{L}{r_{s} \cdot \tau}\right\rceil$. Assume that the time slots along the path are indexed as $1,2, \cdots,|T|$. Let $A(v)$ represent the set of consecutive time slots in which the data transmitted by sensor $v \in V$ can be collected by the mobile sink, which is determined by the maximum transmission range $R_{\max }$ of $v$ and its distance from the sink path. Fig. 1 illustrates such an example, where for two sensors $v_{i}$ and $v_{j}, A\left(v_{i}\right)$ is $\left\{i_{s}, \cdots i_{e}\right\}$ and $A\left(v_{j}\right)$ is $\left\{j_{s}, \cdots j_{e}\right\}$ with $1 \leq i_{s} \leq i_{e} \leq|T|, 1 \leq j_{s} \leq j_{e} \leq|T|$. Notice that $A\left(v_{i}\right) \cap A\left(v_{j}\right) \neq \emptyset$, which means that they share some common time slots at which both of them can transfer their data to the mobile sink. However, following the wireless communication interference model [20], the mobile sink at any given time slot can only receive data from at most one of the sensors.

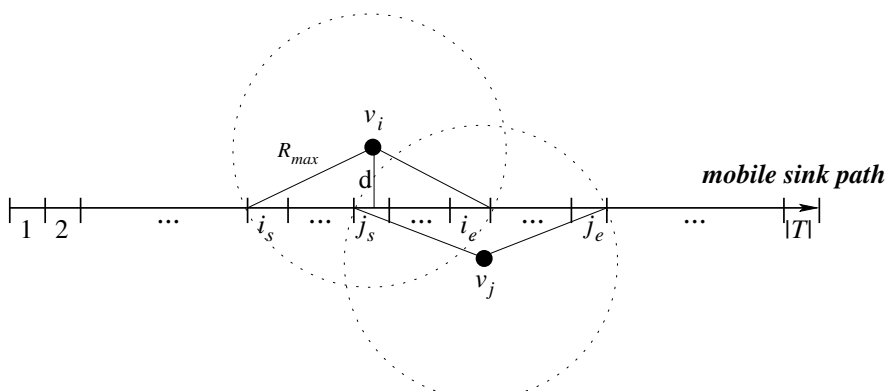

Fig. 1. An illustration of time slots covered by sensors $v_{i}$ and $v_{j}$.

Following Shannon's formula that $C=W \cdot \log \left(\frac{P}{N_{0}}+1\right)$ [17], where $C$ is the channel capacity, $W$ is the bandwidth of the channel, $N_{0}$ is the white noise power, and $P$ is the average transmitter power of the sender which is of the super-linear relationship to the transmitter-receiver distance, the transmission rate is bounded by the channel capacity. Assuming that the transmission power of each sensor $v_{i}, P_{v_{i}}$, is fixed, in this paper a multi-rate communication between $v_{i}$ and $s$ is adopted [7]. That is, the transmission rate of $v_{i}$ is determined by its distance to the receiver (the mobile sink). As shown by Fig. 2, the transmission rates of $v_{i}$ at two different time slots $j$ and $k, r_{i j}$ and $r_{i k}$, are determined by their distances $d_{i j}$ and $d_{i k}$. We thus assume that $r_{i j}$ and $r_{i k}$ are given in the rest of discussion.

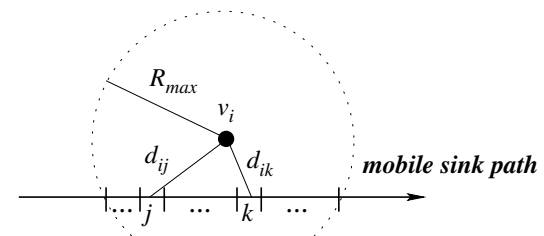

Fig. 2. Multi-rate wireless communication with a fixed transmission power. 


\section{B. Energy model}

We follow a widely adopted assumption of renewable energy replenishment. That is the energy replenishment rate of each sensor is much slower than its energy consumption rate and the amount of harvested energy at a future time period is uncontrollable but predictable based on the source type and harvesting history [10]. Denote by $B_{v}^{\max }$ the energy storage capacity and $B_{v}(i)$ the amount of energy stored at each node $v \in V$ in the beginning of tour $i, B_{v}(i)$ can be expressed as $\min \left\{B_{v}(i-1)+Q_{v}(i-1)-O_{v}(i-1), B_{v}^{\max }\right\}$, where $Q_{v}(i-1)$ and $O_{v}(i-1)$ are the amounts of energy harvested and consumed at tour $i-1$, and $0 \leq B_{v}(i) \leq B_{v}^{\max }$. Furthermore, to support long-period, continuous monitoring service, we assume that sensors should not consume more energy than they can collect in order to achieve perpetual operations [8]. Hence, we use $B_{v}(i)$ as the energy budget of sensor $v$ for tour $i$. Without loss of generality, we define $B_{v}$ as the energy budget of sensor $v$ per tour.

\section{Network utility}

Since the energy replenishment rates vary over time, sensor nodes with sufficient energy replenishment rates may have more chances to transmit their data to the mobile sink, while the others with low energy replenishment rates may never have any chances to transmit their data to the mobile sink at all. Consequently, the data collected per tour is the biased data, which may not represent the data landscape of the entire network. In order to characterize the impact of sensing data from individual sensors on the overall data quality and to achieve proportional fairness among sensors, we introduce a metric, a non-decreasing positive, concave utility function $U(\cdot)$. Denote by $D_{v}$ the amount of data collected from sensor $v$ by the mobile sink per tour, the utility accrued by sensor $v$ is $U\left(D_{v}\right)=\sqrt{D_{v}}$, which is strictly concave and known to achieve proportional fairness [24]. This function reflects an important fact that for any given sensor, when the amount of data collected by the sensor is above a specific threshold, the increase on the utility gain above the threshold is only marginal. The network utility $U_{\text {total }}$ accrued jointly by all sensors per tour thus is:

$$
U_{\text {total }}=\sum_{v \in V} U\left(D_{v}\right)
$$

\section{Problem definition}

Given an energy harvesting sensor network $G$, and a set $T$ of time slots per tour in which the mobile sink can collect data, the network utility maximization problem is to maximize the network utility by allocating the time slots to individual sensors under their energy replenishment rate constraints. Ideally, each sensor should transmit its data at all available time slots to the mobile sink to maximize its utility, thereby maximizing the network utility. However, since the energy replenishment rate of each sensor is much slower than its energy consumption rate, each sensor can only make use of some of these time slots to transmit its data. Furthermore, due to the fact that sensors usually are densely deployed, it is very likely that multiple sensors will compete their shared time slots to transmit their data. Thus, to allocate each shared time slot to which sensor so as to maximize the network utility is a challenging task.

Recall that $A\left(v_{i}\right)$ represents the set of available time slots in which sensor $v_{i}$ can transfer its data, and $r_{i j}$ represents the data transmission rate of sensor $v_{i}$ at time slot $j$. Let

$$
x_{i j}= \begin{cases}1, & \text { time slot } j \text { is allocated to sensor } s_{i} \\ 0, & \text { otherwise }\end{cases}
$$

The network utility maximization problem can be expressed as a non-linear program as follows.

$$
\begin{array}{cc}
\text { Maximize } & U_{\text {total }}=\sum_{v_{i} \in V} U\left(\sum_{j=1}^{|T|} x_{i j} \cdot r_{i j} \cdot \tau\right) \\
\text { Subject to } & x_{i j} \in\{0,1\}, \forall v_{i} \in V, 1 \leq j \leq|T| \\
& x_{i j}=0, \forall v_{i} \in V, j \notin A\left(v_{i}\right) \\
& \sum_{i=1}^{|V|} x_{i j} \leq 1, \forall 1 \leq j \leq|T| \\
& \sum_{j=1}^{|T|} x_{i j} \cdot P_{v_{i}} \cdot \tau \leq B_{v_{i}}, \forall v_{i} \in V
\end{array}
$$

where

- Constraint (5) ensures that at any given time slot, a sensor can transmit its data to the mobile sink only when the sink is within its transmission range.

- Constraint (6) enforces that at most one sensor can transfer its data to the mobile sink if there are multiple such sensors at any given time slot.

- Constraint (7) ensures that the energy consumption of each sensor per tour cannot be exceed its energy budget, where the energy budget $B_{v_{i}}$ of sensor $v_{i}$ is the energy stored in the beginning of this tour, and $P_{v_{i}}$ is the transmission power of sensor $v_{i}$.

\section{E. NP-hardness}

Theorem 1: The network utility maximization problem in an energy harvesting sensor network is NP-hard.

Proof: We show the claim by a reduction from a well known NP-complete problem - the generalized assignment problem (GAP) [4], as follows. An instance of GAP is: let $K=\left\{k_{1}, k_{2}, \cdots k_{n}\right\}$ be a set of jobs, and $F=$ $\left\{f_{1}, f_{2}, \cdots f_{m}\right\}$ a set of agents. Let $B=b_{1}, b_{2}, \ldots, b_{m}$ be the resources capacities, where $b_{i}$ is the capacity of agent $f_{i} \in F$. For each job $k_{j} \in K$ and an agent $f_{i} \in F$, define $b_{i j}$ as the amount of resources required by agent $f_{i}$ to perform job $k_{j}$ and $c_{i j}$ the achieved profit if agent $f_{i}$ is assigned to job $k_{j}$. The optimizing objective is to assign jobs to agents such that the total profit is maximized, subject to the resource capacity constraint at each agent.

We now consider a special case of the network utility maximization problem where we assume that the maximum transmission range of each sensor is large enough to cover the entire tour. That is, a sensor can utilize all time slots per tour. We then perform the reduction, where the set of time slots is the set of jobs, the set of sensors is the set of agents, the energy budget of each sensor is the resource capacity of 
each agent. Moreover, for each time slot $j$ and a sensor $v_{i}$, the energy consumption of sensor $v_{i}$ (the resource consumed) is $b_{i j}=P_{v_{i}} \cdot \tau$ if it transfers its data at the time slot $j$. The utility gain of sensor $v_{i}$ at this time slot is the profit $c_{i j}$. The problem thus is equivalent to GAP. Hence, the network utility maximization problem is NP-hard, too.

\section{Centralized Algorithm}

Since the network utility maximization problem is NP-hard, we deal with the problem by devising a heuristic algorithm, assuming that the mobile sink has a global knowledge of both network topology and the energy information of each sensor (e.g. the energy budget of each sensor). Given the $|T|$ time slots in the tour, the algorithm will assign time slots to sensors. Thus, each sensor can transmit its data to the mobile sinks at the time slots allocated to it. Specifically, the algorithm proceeds iteratively. Within each iteration, one time slot is examined and allocated to a sensor if possible. This procedure continues until all time slots have been examined or allocated. In the following we explain how to examine or allocate a time slot in detail. Given a time slot $j \in T$, denote by $N(j)=\left\{v_{i} \mid j \in A\left(v_{i}\right), B_{v_{i}} \geq P_{v_{i}} \cdot \tau\right\}$ the set of sensors that have enough energy budgets to transfer their data at time slot $j$. If $N(j)$ is $\emptyset$, it implies that no sensor will transmit its data at this slot due to the fact that either all the sensors can not communicate with the mobile sink or they do not have enough energy for data transmission. Otherwise, time slot $j$ is allocated to sensor $v_{i} \in N(j)$, and $v_{i}$ will transfer its data to the mobile sink at time slot $j$, where $v_{i}$ is chosen as follows.

Let $\Delta U\left(v_{i}, j\right)=U\left(D_{v_{i}}^{\prime}+r_{i j} \cdot \tau\right)-U\left(D_{v_{i}}^{\prime}\right)$ be the utility gain of sensor $v_{i}$ by assigning time slot $j$ to it for uploading its data, where $D_{v_{i}}^{\prime}$ is the accumulative amount of data uploaded by $v_{i}$ at the previous $j-1$ time slots in the current tour. To maximize the network utility, we allocate time slot $j$ to the sensor with the maximum utility gain. The detailed algorithm C_Schedule as Algorithm 1 is present as follows.

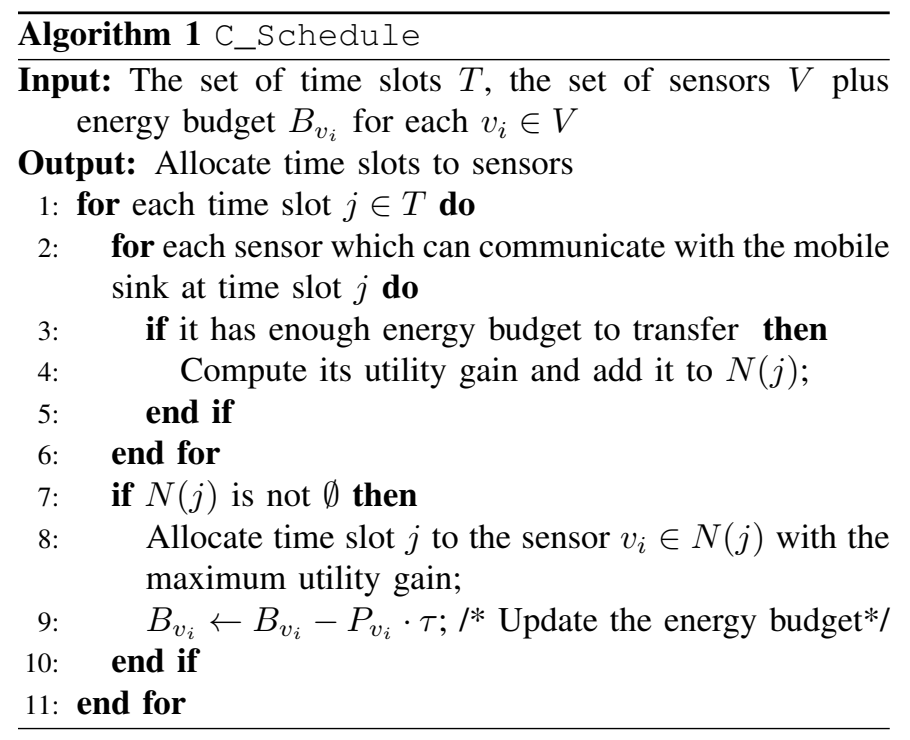

Theorem 2: Given an energy harvesting sensor network $G(V \cup\{s\}, E)$ and a set of time slots $T$, there is an algorithm for the network utility maximization problem, which takes $O(|V| \cdot|T|)$ time.

Proof: We analyze the time complexity of algorithm C_Schedule as follows. Within each iteration, one time slot $j \in T$ will be allocated, which takes $O(|V|)$ time due to the construction of $N(j)$ and finding a node in $N(j)$ with the maximum utility gain. The number of iterations is determined by $|T|$. Hence the algorithm takes $O(|V| \cdot|T|)$ time.

\section{Distributed Algorithm}

In this section, we propose a distributed solution to the problem by removing the assumption that the mobile sink has the global knowledge of the network.

The proposed distributed algorithm proceeds as follows. The mobile sink periodically broadcasts a 'Poll' message with a 'Registration' timer, announcing its presence while traveling along the path. The 'Poll' message is broadcast in the beginning of each interval, where an interval consists of a fixed number of time slots. The 'Poll' message is used to detect whether the mobile sink are within the transmission ranges of the sensors receiving the message. Once a sensor received the 'Poll' message, it acknowledges by sending a 'Registration' message that contains the current energy of the sensor, its data transmission rates at each time slot within this interval, and the accumulative volume of its data uploaded in previous intervals. Once the 'Registration' timer expires, the mobile sink starts assigning the time slots in this interval to the 'Registered' sensors, which essentially is identical to the allocation phase of algorithm 1. The mobile sink then broadcasts a 'Schedule' message which contains the time slot allocation to the registered sensors. Having received the 'Schedule' message, each registered sensor transmits its data to the mobile sink at the time slots allocated to it. The detailed algorithm D_Schedule is described in Algorithm 2.

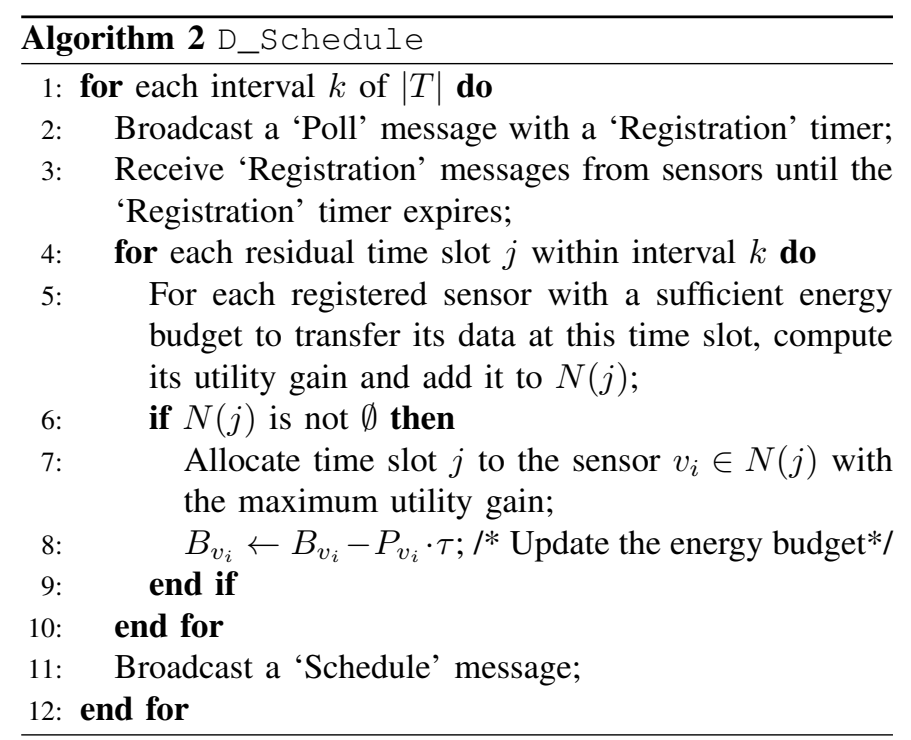




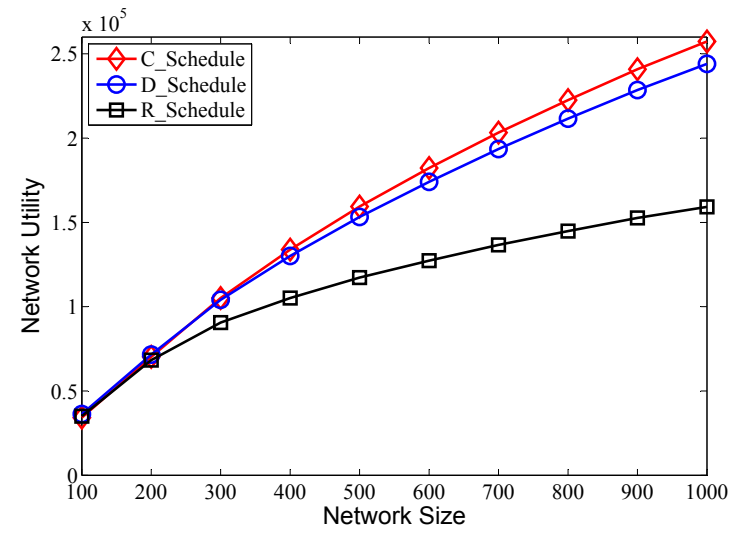

(a) $r_{s}=5 \mathrm{~m} / \mathrm{s}$

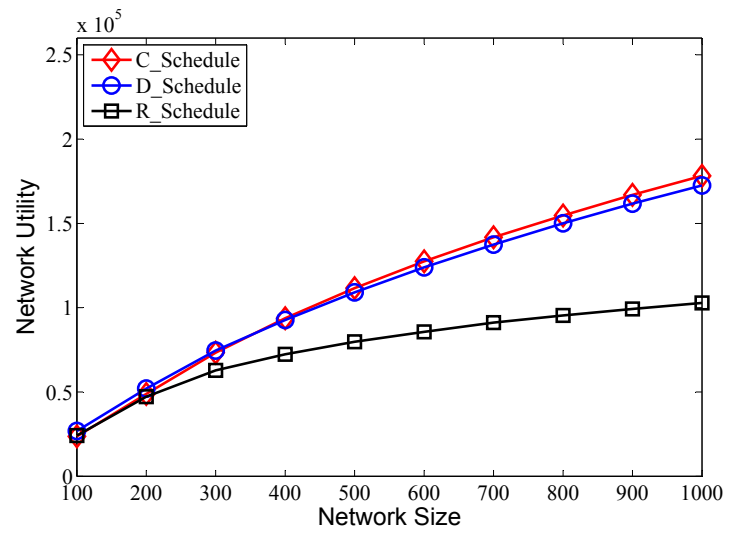

(b) $r_{s}=10 \mathrm{~m} / \mathrm{s}$

Fig. 3. Network utility performance of different algorithms by varying the network size $n$ when the sink speed is $r_{s}=5 \mathrm{~m} / \mathrm{s}$ and $r_{s}=10 \mathrm{~m} / \mathrm{s}$ respectively.

\section{Performance Evaluation}

In this section we study the performance of the proposed algorithms through experimental simulation. We also investigate the impact of parameters: the network size $n$ and the mobile sink speed $r_{s}$ on the performance.

\section{A. Experimental environment setting}

We consider an energy harvesting sensor network consisting of 100 to 1,000 sensors randomly deployed along a given path of a mobile sink, where the path length is $18,000 \mathrm{~m}$ and the maximum distance between the location of any given sensor and the path is $190 \mathrm{~m}$. We assume that all sensors have identical maximum transmission ranges $R_{\max }$ of 200 meters. Each sensor is powered by a $10 \mathrm{~mm} \times 10 \mathrm{~mm}$ square solar panel and its battery capacity is 10,000 Joules. The solar power harvesting profile is built upon the real solar radiation measurements [14], in which the total amount of energy collected from a $37 \mathrm{~mm} \times 37 \mathrm{~mm}$ solar panel over a 48hour period is $655.15 \mathrm{mWh}$ in a sunny day and $313.70 \mathrm{mWh}$ in a partly cloudy day. We here adopt the communication parameters of real radio $C C 2591$ by TI [1], where the energy transmission consumption is $300 \mathrm{~mJ} / \mathrm{s}$, and the available data transmission rates and corresponding distances are: $250 \mathrm{Kbps}$ between 0 and 20 meters, $19.2 \mathrm{Kbps}$ between 20 and 50 meters, $9.6 \mathrm{Kbps}$ between 50 and 120 meters, and $4.8 \mathrm{Kbps}$ between 120 and 200 meters. We set the duration of each time slot $\tau$ is 1 second in the default setting. Each value in figures is the mean of the results by applying each mentioned algorithm to 100 different network topologies of the same network size.

\section{B. Performance evaluation of different algorithms}

We first study the performance of algorithms C_Schedule and D_Schedule against that of another heuristic R_Schedule by varying $n$ from 100 to 1,000 while $r_{s}$ is set at $5 \mathrm{~m} / \mathrm{s}$ and $10 \mathrm{~m} / \mathrm{s}$, respectively, where R_Schedule is a variant of C_Schedule by allocating each time slot $j$ to one sensor in $N(j)$ randomly in each iteration.
Fig. 3 clearly shows that both algorithms C_Schedule and D_Schedule outperform algorithm R_Schedule, and the performance gap between them becomes bigger and bigger with the growth of network size $n$. Specifically, when the network size is 100 and the mobile sink speed is fixed at $10 \mathrm{~m} / \mathrm{s}$, the network utilities of algorithms C_Schedule, D_Schedule, and R_Schedule are almost identical. However, when the network size is 1,000 , the network utility gap between C_Schedule and R_Schedule is no less than $73 \%$, while the network utility gap between D_Schedule and R_Schedule is no less than $67 \%$. In addition, notice that C_Schedule outperforms D_Schedule slightly, as D_Schedule only has the local knowledge rather than the global knowledge of the network and a fractional number of time slots in each interval will be used for sensor detection rather than using for sensing data transmission.

We then investigate the impact of network size $n$ and the mobile sink speed $r_{s}$ on the network utility, by varying $n$ from 100 to 1,000 and $r_{s}$ from $5 \mathrm{~m} / \mathrm{s}$ to $20 \mathrm{~m} / \mathrm{s}$, respectively.

Fig. 4 indicates that with the decrease of $r_{s}$, the network utilities delivered by algorithms C_Schedule, D_Schedule, and R_Schedule increase. Specifically, the network utility delivered by each mentioned algorithm when $r_{s}=5 \mathrm{~m} / \mathrm{s}$ is at least $43 \%, 77 \%$, and $108 \%$ higher than that by itself when $r_{s}=10 \mathrm{~m} / \mathrm{s}, 15 \mathrm{~m} / \mathrm{s}$, and $20 \mathrm{~m} / \mathrm{s}$, respectively. This is because when the mobile sink reduces its traveling speed, the sensors will have more time slots available for their data uploading. This will lead to longer delay on data delivery. We also notice that with the increase of network size, the network utilities of all mentioned algorithms increase, too.

\section{CONCLUSION AND FUtURE WORK}

In this paper we studied mobile data collection in an energy harvesting sensor network with a path-constrained mobile sink. We first formulated the problem as a novel network utility maximization problem and showed that the problem is NP-hard. We then devised a centralized algorithm, assuming 


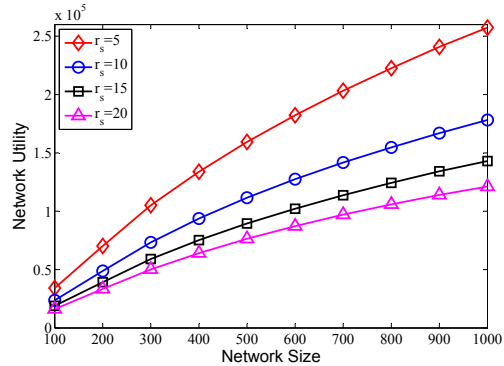

(a) Algorithm C_Schedule

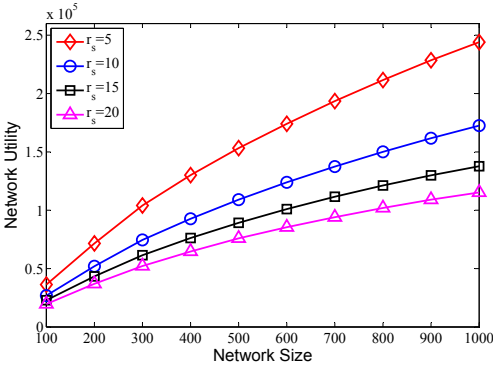

(b) Algorithm D_Schedule

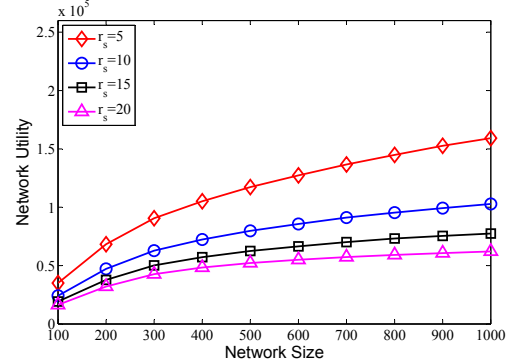

(c) Algorithm R_Schedule

Fig. 4. Impact of parameters $n$ and $r_{s}$ on the network utilities delivered by mentioned algorithms.

that the global knowledge of the entire network is available. We also proposed a distributed algorithm without the global knowledge of the network. Finally, we conducted experiments by simulations to evaluate the performance of the proposed algorithms, and the experimental results demonstrate that the proposed algorithms are efficient.

\section{REFERENCES}

[1] CC2591 RF front end. available at http://www.ti.com.

[2] A. Chakrabarti, A. Sabharwal, and B. Aazhang. Communication power optimization in a sensor network with a path-constrained mobile observer. ACM Trans. Sen. Netw., 2(3):297-324, August 2006.

[3] M. D. Francesco, S. K. Das, and G. Anastasi. Data collection in wireless sensor networks with mobile elements: A survey. ACM Trans. Sen. Netw., 8(1):7:1-7:31, August 2011.

[4] M. L. Fisher, R. Jaikumar, and L.-N. Wassenhove. A multiplier adjustment method for the generalized assignment problem. Manage. Sci., 32(9):1095-1103, September 1986.

[5] K.-W. Fan, Z. Zheng, and P. Sinha. Steady and fair rate allocation for rechargeable sensors in perpetual sensor networks. In Proc. of SenSys'08, ACM, 2008.

[6] S. Gao, H .Zhang, and S. K. Das. Efficient data collection in wireless sensor networks with path-constrained mobile sinks. IEEE Trans. Mobi. Comput., 10:592-608, 2011.

[7] L. He, J. Pan, and J. Xu. A progressive approach to reducing data collection latency in wireless sensor networks with mobile elements. IEEE Trans. Mobi. Comput., 99(PrePrints), 2012.

[8] A. Kansal, J. Hsu, S. Zahedi, and M. B. Srivastava. Power management in energy harvesting sensor networks. ACM Trans. Embed. Comput. Syst., 6(4):32, September 2007.

[9] A. Kansal, A. A. Somasundara, D. D. Jea, M. B. Srivastava, and D. Estrin. Intelligent fluid infrastructure for embedded networks. In Proc. of MobiSys'04, ACM, 2004.

[10] R.-S. Liu, K.-W. Fan, Z. Zheng, and P. Sinha. Perpetual and fair data collection for environmental energy harvesting sensor networks. IEEE/ACM Trans. Netw., 19(4):947-960, August 2011.

[11] W. Liang, J. Luo, and X. Xu. Prolonging network lifetime via a controlled mobile sink in wireless sensor networks. In Proc. of Globecom'10, IEEE, 2010.

[12] W. Liang and J. Luo. Network lifetime maximization in sensor networks with multiple mobile sinks. In Proc. of LCN'11, IEEE, 2011.

[13] W. Liang, P. Schweitzer, and Z. Xu. Approximation algorithms for capacitated minimum spanning forest problems in wireless sensor networks with a mobile sink. To appear in IEEE Trans. on Computers, June, 2012.

[14] R.-S. Liu, P. Sinha, and C. E. Koksal. Joint energy management and resource allocation in rechargeable sensor networks. In Proc. of INFOCOM'10, IEEE, 2010.

[15] L. Lin, N. B. Shroff, and R. Srikant. Asymptotically optimal energyaware routing for multihop wireless networks with renewable energy sources. IEEE/ACM Trans. Netw., 15(5), October 2007.

[16] X. Ren, and W. Liang. Delay-tolerant data gathering in energy harvesting sensor networks with a mobile sink. To appear in Proc. of GLOBECOM'12, IEEE, 2012.
[17] C. E. Shannon. A mathematical theory of communication. The Bell System Technical Journal, 27:379-423, 623-656, October 1948.

[18] L. Song and D. Hatzinakos. Architecture of wireless sensor networks with mobile sinks: sparsely deployed sensors. IEEE Tran. Vehi. Tech., 56(4):1826-1836, July 2007.

[19] A. A. Somasundara, A. Kansal, D. D. Jea, D. Estrin, and M. B. Srivastava. Controllably mobile infrastructure for low energy embedded networks. IEEE Trans. Mobi. Comput., 5(8):958-973, August 2006.

[20] Q. Wang, D. O. Wu, and P. Fan. Delay-constrained optimal link scheduling in wireless sensor networks. IEEE Tran. Vehi. Tech., 59(9):4564-4577, November 2010.

[21] X. Xu and W. Liang. Monitoring quality optimization in wireless sensor networks with a mobile sink. In Proc. of MSWiM'11, ACM, 2011.

[22] X. Xu, W. Liang, and T. Wark. Data quality maximization in sensor networks with a mobile sink. In Proc. of DCOSS'11, IEEE, 2011.

[23] Z. Xu, W. Liang, and Y. Xu. Network lifetime maximization in delaytolerant sensor networks with a mobile sink. In Proc. of DCOSS'12, IEEE, 2012.

[24] B. Zhang, R. Simon, and H. Aydin. Maximum utility rate allocation for energy harvesting wireless sensor networks. In Proc. of MSWiM'11, ACM, 2011. 